\title{
Driving the Initiative of Service-Oriented Architecture Implementation
}

\author{
Muhammad Suhaizan Sulong ${ }^{1,2}$, Andy Koronios ${ }^{2}$, Jing $\mathrm{Gao}^{2}$ \\ and Azlianor Abdul-Aziz ${ }^{1,2}$ \\ ${ }^{1}$ Department of Software Engineering, Universiti Teknikal Malaysia Melaka, Malaysia \\ ${ }^{2}$ Strategic Information Management Research Group, University of South Australia, Australia
}

\begin{abstract}
Over the years, many organisations have invested heavily in information systems, changing the way they do business for improvement. Today however, aligning business and information systems is not easily achieved due to rapidly changing business environment. The ability of an organisation to change and change quickly in response to business needs is of paramount concern. This means the current traditional approach of adopting information systems is not suitable. Thus, a need of new paradigm to make business more agile and flexible is undeniable. The new paradigm is that taking a service-oriented architecture initiative as an enabling technology for critical business processes. This review paper has identified a distinctive approach compared to traditional information systems, a generic initiative process as well as the success factors of implementing the service-oriented architecture initiative. Further work is required through case study to obtain insightful understanding on any related issues and challenges in order to build better solutions for such initiative.
\end{abstract}

Keywords: Service-Oriented Architecture, Information Systems, Success Factors.

\section{Introduction}

In this new era of information technology, any organisation that seeks to improve business performance needs to transform their traditional business to be more efficient and collaborative. Implementing a new way of doing business is just another way that successful organisations can easily grow their business fast. There are two ways for leveraging information technology for business benefit: implementing SOA initiative or adopting information systems that both require information technology to support its successful outcome. It is important to look at the characteristics and capabilities of SOA and information systems before an organisation choosing to adopt. For that reason, the uniqueness of SOA compared to information systems can be recognised where it depends on what they can provide to improve business performance throughout the business lifetime.

Information systems and SOA are two closely related technologies, but conceptually they are different. Information system refers to an interconnected of computer hardware, software, data, procedures and users that interact to produce information in order to help businesses achieve their goals and objectives (Kroenke, 2010); whereas SOA is a design philosophy describing an architecture of implementing information technology within an organisation (Selamat \& Al Kharusi, 2009). Most organisations

Copyright (C) 2012 Muhammad Suhaizan Sulong, Andy Koronios, Jing Gao and Azlianor Abdul-Aziz. This is an open access article distributed under the Creative Commons Attribution License unported 3.0, which permits unrestricted use, distribution, and reproduction in any medium, provided that original work is properly cited. Contact author: Muhammad Suhaizan Sulong E-mail: MuhammadSuhaizan.Sulong@ postgrads.unisa.edu.au 
with information systems always find themselves trying to cope with business changes that make the establishment of business objectives and priorities difficult (Salmela \& Spil, 2002). That is why not only the information systems that need to adopt but also an effective business process solution of meeting constantly changing business environment is needed (Kaipia et al., 2002).

Change will always happen in the organisations when there are new opportunities and new businesses required. Thus, seeking agility and flexibility is the key importance to meet new market demands. Many organisations have foresee this importance and wanted not to abandon their existing legacy systems and information technology infrastructure (Guah, 2009). Organisations should therefore have the initiatives to implement SOA which will vastly increase the overall benefits - increase productivity, better services and faster delivery (Bastida et al., 2008). This means SOA initiative implementation provides business processes that are shared and integrated services in order to achieve its agility and flexibility. Implementing SOA initiatives gives a huge advantage compared to adopting information systems.

This paper is structured as follows: the first section describes the SOA initiative and process of implementing such initiative via phases. The second section discusses the factors consideration for implementing SOA initiatives. Finally, the paper describes a method for further work through case studies and draws a conclusion.

\section{Research Method}

This paper analyses SOA related literature on how organisations initiate the implementation of SOA across their departments. The method for analysing the insight from the literature consists of four steps as illustrated in Fig 1.
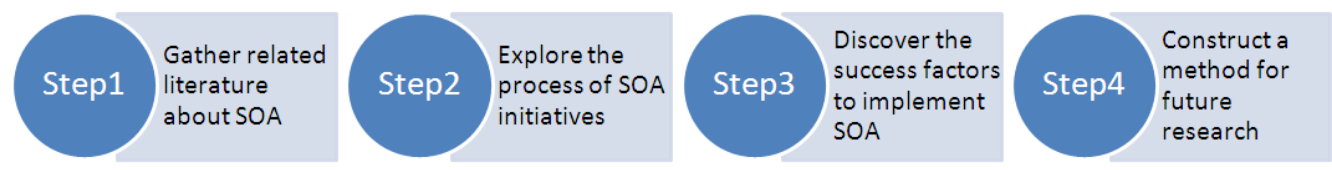

Fig 1. Steps Analysing the Literature (Developed for This Study)

In the first step, the literature about SOA, from academic proceedings and journals, especially relevant to the organisations initiative in implementing SOA is gathered. These proceedings and journal articles were classified and organised to seek SOA initiative process and factors consideration of SOA for organisations to implement SOA initiative. Besides articles, the white papers from industrial research have also been considered. Then in step 2, the generic process of implementing SOA initiative into an organisation is explored and determined. In step 3, the success factors to implement SOA that are considered by organisations were discovered. In the last step, the basis from this literature will be taken to further research through conducting case study using semistructured interview.

\section{Distinctive Service-Oriented Architecture Initiatives}

SOA is critically the way to moving to the next era of business computing with web services that can be easily integrated within legacy systems as well as existing information system applications and of course, are compatible with current business processes and practices (Chen, Sen \& Shao, 2006). Obviously, SOA involves a change in information systems approach as in Table 1 that shows the key differences between SOA and information systems. 
Table 1: Differences between SOA and Information Systems

\begin{tabular}{|l|l|}
\hline \multicolumn{1}{|c|}{ Service-Oriented Architecture } & \multicolumn{1}{c|}{ Information Systems } \\
\hline Loose coupling (Russel et al., 2006) & Tight coupling (Sutherland \& Heuvel, 2002) \\
\hline Services (Erl, 2005) & Processes (Middleton, 2007) \\
\hline No redundancy (Schelp \& Schwinn, 2005) & Redundancy (El Ouedghiri, 2007) \\
\hline Ease of integration (Josuttis, 2007) & Complex integration (Giachetti, 2004) \\
\hline Agile methodology (Bloomberg, 2003) & Traditional methodology (Barry \& Lang, 2003) \\
\hline Source: (developed for this study) &
\end{tabular}

It is clear that SOA implements business processes as services where it encapsulates the data and functions that makes it a loosely coupled - flexible use of services across organisational boundaries (Erl, 2005; Russel et al., 2006); that means the services are accessible by different and multiple users. On the other hand, in information systems, it emphasises the systems and processes which is in static implementation and it does not allow to be adapted to changes as business grows, and thus it is tightly coupled (Sutherland \& Heuvel, 2002; Middleton, 2007). While information systems use traditional methodology, for example the famous SSADM - structured systems analysis and design method (Barry \& Lang 2003), agile methodology is most suitably used in the SOA development; for example, extreme programming (XP) methodology, that enables SOA to be agile by easily and simply changing and adding new services according to the changing business environment (Bloomberg, 2003).

Moreover, organisations that have implemented a vast numbers of information systems across organisations tend to produce redundant information that is complex to integrate which can make tasks complicated for business users to consolidate this information (Giachetti, 2004; El Ouedghiri, 2007). However, in SOA system, the development of services that involve technical and business perspective results in no redundancy (Schelp \& Schwinn, 2005), and thus it allows easy integration without changing the legacy systems (Josuttis, 2007).
Taking into account these differences between SOA and information systems, it can be said that implementing SOA initiative is far-beyond the scope of adopting generic information systems that offer a great value to organisations.

\section{Service-Oriented Architecture Initiative Phases}

It must be pointed out that there are many approaches to implement the SOA initiative in an organisation. The approaches can be a consulting-driven approach or technologydriven approach from SOA providers. Consulting-driven approach ensures deep process understanding for delivering right SOA strategy for an organisation and implementing SOA best practices for gaining optimum results. Technologydriven approach, on the other hand, focuses on information technology to implementing SOA initiative in which the SOA strategy, services and infrastructure are developed based on that technology.

Forrester researchers have evaluated SOA providers and found out six leading SOA providers that are Accenture, Software AG, TIBCO, IBM, SAP and Oracle (Heffner et al., 2008; Vollmer et al., 2008). These providers offer different ways of implementing SOA initiatives and the approaches were reviewed and compared. Consequently, four phases were derived: strategic planning, building architecture, services operation and continuous improvement as illustrated in Fig 2. 


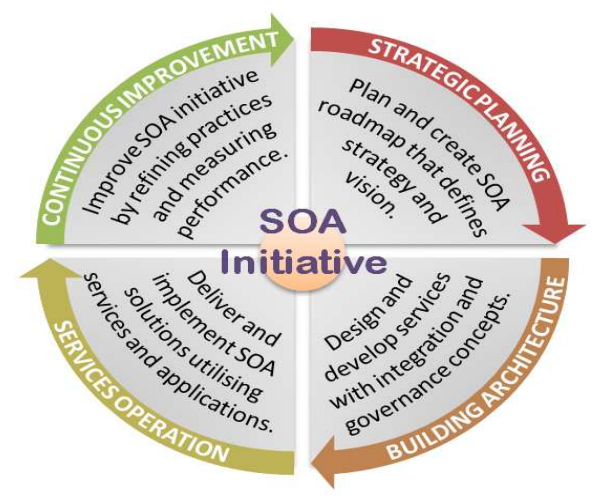

Fig 2. Four Phases of SOA Initiative Process (Developed for This Study)

Strategic Planning - The main focus in this phase is to plan and create SOA roadmap that defines strategy and vision which places emphasis on balancing the non-technical and technical elements for implementing SOA initiative (Hack \& Lindemann, 2008). Gandhi (2009) lists some of the areas that require strategic planning while implementing an SOA approach, namely, service monitoring (including audits, SLAs and performance management), exception management (including root cause analysis), version management, service management and deployment, policy and security considerations, service level agreements, service directory (including service life cycle methodology). Moreover, a thorough formulation of business objectives and information technology must be established for an organisation to obtain value from a SOA implementation.

Building Architecture - The core process in this phase is the design and development of SOA services with integration and governance concept that can scale seamlessly across an organisation. One example of this SOA service is web services where through web services technology, SOA can be implemented, with new and existing information systems as functional building blocks (Karande et al., 2011). This web services can then be integrated with other web services to fulfil the organisation's business requirements. In addition, IBM (2007) recommends that this is the right stage for training staff and preparing the organisation to adopt the SOA framework in order to ensure that they understand and therefore will accept the new model with greater ease.

Services Operation - Once the web services are developed, integrated and tested, they are delivered to the marketplace to allow consumers to utilise the range of web services in their business (Accenture, 2007). An operational guideline is required to ensure continuous operation and support for all the available services and to enable the integration and the information to flow freely between the various systems and applications (Barry, 2003). The web services are operated on dedicated servers within the SOA infrastructure in a controlled environment between organisations and partners. Operating web services include implementation of SOA infrastructure, integration of web services with existing systems and monitoring system performance (Crimson, 2011).

Continuous Improvement - During this phase, a SOA initiative is continuously improved by refining practices and measuring performance. Refining practices is where the challenge is, identifying all necessary areas in the SOA environment that need to improve including standards and guidelines as well as web services to reflect the changes in business processes whereas measuring performance is include assessment of business performance through key performance index analysis and calculation of return on investment (Brown \& Meyer, 2006). In order to continuously improve business processes and optimise costs, planning and operation 
of SOA should closely coordinate to maintain and sustain the implementation of SOA initiative (Jensen \& Basrai, 2010).

\section{Before and After Implementation of Service-Oriented Initiatives Architecture}

Organisations adopting information system can be said that they are more likely in the environment before SOA where applications are dependent on business functions (CA, 2007). This means that these information system applications and databases could be from more than one vendor and located variously in networks and that traditional information technology infrastructure are siloed, non-integrated, closed, monolithic, brittle and vulnerable (Oracle, n.d.). These make them independent and incompatible systems and would have then caused business users face difficulties in performing specific business tasks. That is on one hand before the implementation of SOA.

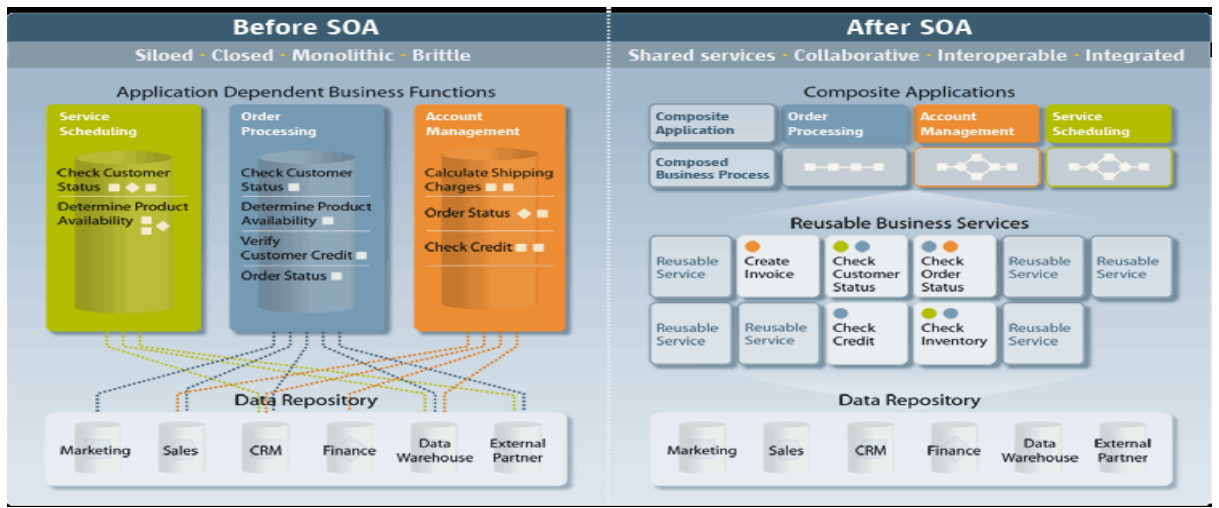

Fig 3. Before and After SOA (Adopted from Oracle, n.d.)

On the other hand, organisations after SOA implementation means that they have become SOA-enabled organisations which have benefited the agility and flexibility of SOA in order to stay competitive in the challenging business environment. This means that business process activities within SOA-enabled organisations are shared services, collaborative, interoperable and integrated in order to deliver new products much faster and compete more effectively in today's changing marketplace (Oracle, n.d.). In addition, the composite applications and reusable business services allow organisations to anticipate faster today's business needs with tomorrow's information technology (Shah, 2007).

\section{Key Success Factors for Implementing Service-Oriented Architecture Initiatives}

Many research-works that study the implementation of SOA initiatives have discovered a number of factors for consideration to successfully implement such initiatives. Consequently, after much deliberate review and analysis, five key classifications of success factors were derived. These factors are discussed next.

\section{Management Support and Strategy Focus}

It is particularly important to have support from top management in every activity in terms of implementing SOA initiative (Josuttis, 2007; Lee, Shim \& Kim, 2010). A strong sponsorship of CEO/CIO and active participation of top managers is vital to successfully implement SOA initiative implementation in order to gain agility, efficiency and flexibility benefits (Lawler et al., 2009). Understanding SOA in the aspect of not only information technology but also business process in which SOA offers a way of structuring a business as a set of services. Without governance and support, the effort of implementing successful SOA initiative can be frustrated (Grigoriu, 2009). 
With all the support that the organisation can get to implement SOA initiative, the focus to ensure SOA implementation should be based on strategy and planning as well as management. A roadmap of stepby-step execution and the scope of technology and applications are determined to emphasise the process structure of operational implementation including the assessment of services performance (Lee, Shim \& Kim, 2010). In addition, for the first time, the experience of introducing SOA initiative, a vigilant approach to establish the best way to apply the SOA technology includes application framework and infrastructure that correspond to the designed SOA architecture, is appropriate (Josuttis, 2007).

\section{Information Quality Management, Standards and Policies}

Today, information management in general and information quality in particular are the most significant factors that organisations must give much attention to (TIBCO, 2007). This is to ensure data integrity and quality for technical and nontechnical functions to provide a higher quality output of SOA services and applications once they are operated (Lawler et al., 2009; Morgenthal, 2009). Furthermore, Rice (2009) presents some attributes of quality such as correctness, performance, security, interoperability, usability, maintainability, reliability and portability in order to test SOA services successfully.

Every service and application in SOA architecture implementation needs to follow certain rules and appropriate standards in order to fulfil specific needs of service consumers (Wesemann, 2006). The standards and policies allow the services operated according to specifications and meet business goals which constitute a means to effective control of the service portfolio (Macehiter \& Ward-Dutton, 2005). In this way, the appropriate level of quality that the services operate can be enforced and consistent across SOA operations.

\section{Business Relationship and Communication}

The relationship with services user and service provider is necessary, in which it is clearly the most challenging part of SOA initiatives to establish change management and ease the provisioning of services throughout the SOA life cycle (Mulholland, Daniels \& Hall, 2008). In addition, the support of key business stakeholders is important to have a good business relationship as well as a certain long-term participation (Wesemann, 2006). This means that these stakeholders contribute to the overall needs of the organisation and support its goals in order to sustain longterm effort of SOA journey.

Designated SOA team should be in place and are keen to successfully implement SOA initiatives (Josuttis, 2007). This team may consist of business experts and/or business analysts that are capable of providing decisions around designing business solutions across SOA complex layers (Lee, Shim \& Kim, 2010). Executive business and technology leadership both are vital in which their roles and responsibilities in the SOA program are clearly defined for completing SOA initiatives journey in the aspect of critical business and technical SOA strategies (Lawler et al., 2009).

Successful communication proves necessary as one of the major factors towards enabling the SOA initiative implementation in which business and IT personnel discuss about policies, protocols and standards with key stakeholders (Rogers, 2008). In every activity at each phase of SOA initiative, communication is vital, and a more powerful communication channel is required in order to reduce the complexity of SOA services (Lee, Shim \& Kim, 2010). For instance, with effective communication method between service designers and consumers, the difficulties in handling problems and issues would be resolved and devised. 


\section{Architecture Design and Implementation}

Development methodology for SOA services is different from traditional methodology in terms of the approach in building services. There are two approaches that utilise the overall development as a strategy: 1) top-down approach - dividing the existing system into components; and 2) bottom-up approach - extracting events from business process (Lee, Shim \& Kim, 2010). Having such approaches in place as a guideline in implementing SOA initiatives according to architecture and development patterns can be useful and clearly show the scalability of the SOA infrastructure architecture with a vision for future expansion (Lawler et al., 2009; IBM, 2008).

Factors included in this classification are related to business process and design where it involves designing SOA services according to business specification of processes, technology-agnostic business design and contractual-like interaction between business and information technology (Grigoriu, 2009). Moreover, factors such as the knowledge of business processes, tools that support processes modelling in terms of interconnecting functional processes and analysis tools enabling modelling principles, are able to bridge business and information technology gap (Ricken \& Petit, 2009).

In order to address information technology alignment to business and strategy, the implementation aspect for operating SOA services towards a single integration system should not be taken lightly. The changes that SOA brings to the current development and operation processes can maximise the usage of existing services and support continuous service monitoring and life cycle (Lee, Shim \& Kim, 2010). It is also important to note that by considering the aspect of implementing and operating services, SOA enables the visibility of operational services through service management and governance (IBM, 2008), untangles the interconnecting services as well as reduces pressure to integrate or replace legacy systems and applications (Grigoriu, 2009).

\section{Change Management and Performance Monitoring}

SOA provides changes to business process and services for agility and flexibility through change management which is a set of procedures that help organisations to ensuring optimal resolution of change requests (Lawler et al., 2009). Besides, the organisational change management that includes culture of innovation, knowledge exchange and collaboration work, are able to create organisational culture and help technical and non-technical employees to embrace the SOA program (Rogers, 2008; Josuttis, 2007).

Measuring performance is essential to keep the services operated continuously by having a mechanism for performance measurement in terms of service reuse and redesign development (Lee, Shim \& Kim, 2010). In addition to that, in order to get efficient business process and continuous process improvement, the performance measurement tool should be devised so that SOA services can operate on a continuous basis to fulfil customer demand in a competitive market (Lawler et al., 2009).

\section{Conclusion and Future Work}

This research will further study the realm of information systems in general and service-oriented architecture in particular, attempts to construct how people work, how technology is used and how organisations operate in association with implementing SOA initiatives. It also aims to obtain in-depth understanding of the business processes and their requirements and to investigate related issues and challenges through accessing the subjective meanings from different perspectives of professionals within natural environments in different organisations. Hence, the interpretive approach through a case study is appropriate for this study (Yin, 2009) and will help obtain qualitative data by applying a qualitative approach through semi-structured interviews (Myer \& Newman, 2007). 
Organisations that have been identified by using information technology when doing business and having deployed SOA are appropriate to be selected as case studies. These organisations might have the potential of having initiative in implementing SOA to improve their business agility and flexibility. The selection is based on opportunistic basis and therefore, most probably would be in Australia. Organisations can be from various industries such as finance, retail, public, utility and health services. The study requires voluntary participants that could provide information regarding the adoption and implementation of SOA initiative. Potential participants including all relevant SOA professionals such as managers, architects and developers have both an understanding of business and information technology involved in implementing SOA initiative.

The paper focuses on the importance of SOA initiative over traditional information system adoption, the implementation phases of SOA initiative - strategic planning, building architecture, services operation and continuous improvement and the five key factors consideration for SOA initiative towards becoming an SOAenabled organisation. This paper will act as a foundation to provide a solid basis for further work to investigate any related issues and challenges that are encountered by organisations that have started or have implemented SOA initiative through conducting case studies. The value of SOA should be taken seriously by organisations wishing to implement SOA initiative in order to improve business agility and flexibility, that are able to cope with business changes and more importantly able to benefit the return on investment over time.

\section{Acknowledgement}

This research is funded by the Ministry of Higher Education Malaysia (MOHE), Universiti Teknikal Malaysia Melaka (UTeM) and University of South Australia (UniSA). We gratefully acknowledge their generous financial support.

\section{References}

Accenture (2007). "Key Stages of the Service Oriented Architecture Journey," Accenture SOA Points of View, Research \& Insight, Accenture. [Online], [Retrieved December 4, 2011], http://www.accenture.com/Global/Techn ology/Service_oriented_Architecture/KeyJ ourney.htm

Barry, C. \& Lang, M. (2003). “A Comparison of 'Traditional' and Multimedia Information Systems Development Practices," Information and Software Technology, 45 (4), 217-227.

Barry, D. K. (2003). Web Services and Service-Oriented Architecture: The Savvy Manager's Guide, Elsevier, USA.

Bastida, L., Berreteaga, A. \& Cañadas, I. (2008). Adopting Service Oriented Architectures Made Simple, Enterprise Interoperability III: New Challenges and Industrial Approaches, Mertins, K., Ruggaber, R., Popplewell, L. \& Xu, X. (ed), Springer London, 221-232.

Bloomberg, J. (2003). "The Role of the Service-Oriented Architect," The Rational Edge. [Online], [Retrieved January 17, 2012],

http://www.therationaledge.com/may_03 /f_bloomberg.jsp.

Brown, P. \& Meyer, R. (2006). “A Planning Guide to SOA," Webinar, TIBCO Software Inc. [Online], [Retrieved January 15, 2012], http://media.tibco.com/video/tibco_1116 06/index.html

CA (2007). SOA and Web Services - The Performance Paradox, White Paper, CA Wily Technology.

Chen, A. N. K., Sen, S. \& Shao, B. B. M. (2006). "Strategies for Effective Web Services Adoption for Dynamic EBusinesses," Decision Support Systems, 42 (2), 789-809. 
Crimson (2011). Oracle SOA vs. IBM SOA: Customer Perspectives on Evaluating Complexity and Business Value, Business White Paper, Crimson Consulting Group.

El Ouedghiri, M. (2007). "AUI Information System Urbanization," in Fourth Annual Meeting American International Consortium of Academic Libraries, Morocco.

Erl, T. (2005). Service-Oriented Architecture: Concepts, Technology, and Design, Prentice Hall, NJ.

Gandhi, N. (2009). "E-Guide SOA Implementation: An SOA Practices Checklist for Implementation Roadmaps," SearchSOA.com, [Online], [Retrieved January 4, 2012], http://media.techtarget.com/Syndication/ STORAGE/Oracle_eGuide_ImplementingSO A.pdf

Giachetti, R. E. (2004). "A Framework to Review the Information Integration of the Enterprise," International Journal of Production Research, 42 (6), 1147-1166.

Grigoriu, A. (2009). 'Enterprise SOA Critical-Success Factors,' The Architecture Journal, 21, 18.

Guah, M. (2009). Managing Very Large IT Projects in Businesses and Organisations, IGI Global, UK.

Hack, S. \& Lindemann, M. A. (2008). Enterprise SOA roadmap. 1st edn, SAP Press.

Heffner, R., Leganza, G. \& Ranade, K. (2008). SOA Adoption: Technology Diversity Accelerators, Forrester Research.

IBM (2007). "Six Critical IT Operations Questions for a Successful SOA Deployment," IBM Global Technology Services, [Online], [Retrieved January 4, 2012], http://www935.ibm.com/services/us/its/pdf/wp_sixcritical-it-operations-questions.pdf.

IBM (2008). "Reaping The Rewards of Your Service-Oriented Architecture
Infrastructure," IBM Global Services, [Online], [Retrieved January 4, 2012], http://www-

935.ibm.com/services/us/cio/pdf/iaw030 02usen.pdf.

Jensen, C. T. \& Basrai, J. (2010). "Continuous Improvement with BPM and Enterprise Architecture Together," Round Tables Online, IBM Corporation, [Online], [Retrieved January 17, 2012], http://www.soainstitute.org/roundtables/past-round-table/article/applyingworld-class-continuous-processimprovement-to-

lower-supply-chain-costs.html.

Josuttis, N. (2007). SOA in Practice, First Edition, O’Reilly Media.

Kaipia, R., Holmström, J. \& Tanskanen, K. (2002). "VMI, What Are You Losing if You Let Your Customer Place Orders?," Journal of Production Planning \& Control, 13 (1), 17-25.

Karande, A. M., Chunekar, V. N., \& Meshram, B. B. (2011). "Working of Web Services Using BPEL Workflow in SOA," Advances in Computing, Communication and Control, Springer Berlin Heidelberg, 125, 143-149.

Kroenke, D. (2010). Experiencing MIS, Prentice Hall, USA.

Lawler, J. P., Benedict, V., Howell-Barber, H.\& Joseph, A. (2009). "Critical Success Factors in the Planning of a ServiceOriented Architecture (SOA) Strategy for Educators and Managers," Information Systems Education Journal, 94 (7).

Lee, J. H., Shim, H.- J. \& Kim, K. K. (2010). "Critical Success Factors in SOA Implementation: An Exploratory Study," Information Systems Management, 27, 123145.

Macehiter, N. \& Ward-Dutton, N. (2005). "Real SOA - Critical Success Factors, Independent Report," Systinet Corporation, [Online], [Retrieved January 15, 2012], http://www.immagic.com/eLibrary/ARCH IVES/GENERAL/SYSTINET/S050617M.pdf. 
Middleton, M. (2007). "A framework for Information Management: Using Case Studies to Test Application," International Journal of Information Management, 27 (1), 9-21.

Morgenthal, J. P. (2009). "Determining SOA Success," Presentation Slide, Morghenthal, [Online], [Retrieved January 15, 2012], http://www.bta.mil/products/BusOpsTran sformation_EA_BI/soa_pdfs/4_T2B_JP\%20 Morgenthal_For\%20Publication.pdf.

Mulholland, A., Daniels, R. \& Hall, T. (2008). "The Cloud and SOA: Creating an Architecture for Today and for the Future," Capgemini, [Online], [Retrieved January 15, 2012],

http://www.hp.com/hpinfo/analystrelatio ns/wp_cloudcomputing_soa_capgemini_hp. pdf

Myers, M. D. \& Newman, M. (2007). "The Qualitative Interview in IS Research: Examining the Craft," Information and Organization, 17, 2-26.

Oracle n.d. "Respond to a Changing Landscape: Service-Oriented Architecture," Oracle Corporation, [Online], [Retrieved January 17, 2012], http://cs.sun.com/practice/software/soa/.

Rice, R. W. (2009). "A Strategy for Testing SOA," Rice Consulting, [Online],[Retrieved January 15, 2012], http://www.riceconsulting.com/articles/S OA-testing-strategy.html.

Ricken, J. \& Petit, M. (2009). "Characterization of Methods for ProcessOriented Engineering of SOA," Business Process Management Workshops, SpringerVerlag, Berlin, 17 (8), 621-632.

Rogers, S. (2008). 'A study in critical success factors for SOA,' Technical report, Hewlett-Packard.

Russell, D. J., Looker, N. \& Xu, J. (2006). "SOA, Dependability, and Measures and Metrics for Network Enabled Capability," in IET Forum on Capability Engineering, London, UK.
Salmela, H. \& Spil, T. A. M. (2002). "Dynamic and Emergent Information Systems Strategy Formulation and Implementation," International Journal of Information Management, 22 (6), 441-460.

Schelp, J. \& Schwinn, A. (2005). "Extending the Business Engineering Framework for Application Integration Purposes," Proceedings of the 2005 ACM Symposium on Applied Computing, ACM Press, New York, 1333-1337.

Selamat, M. H. \& Al Kharusi, A. (2009). "Service Oriented Architecture in Education Sector," International Journal of Computer Science and Network Security, 9 (5), 301-308.

Shah, M. (2007). "SOA reusability: Shrinking the Lag Between Business and IT," Java.net, [Online], [Retrieved January 17 , 2012], http://today.java.net/pub/a/today/2007/ $07 / 24$ /soa-reusability-shrinking-lagtime.html.

Sutherland, J. \& van den Heuvel, W.- J. (2002). "Enterprise Application Integration and Complex Adaptive Systems," Communications of the ACM, 45 (10), 59-64.

TIBCO (2007). "Accurate Data - A Critical Success Factor for SOA," CIO2CIO Perspectives, [Online], [Retrieved January 15 , 2012], http://www.tibco.es/multimedia/wpaccurate-data-a-critical-success-factor-forsoa_tcm55-2417.pdf.

Vollmer, K., Leganza, G., Smillie, K. \& An, M. (2008). The Forrester Wave ${ }^{\mathrm{TM}}$ : IntegrationCentric Business Process Management Suites, Forrester Research, October.

Wesemann, D. (2006). "How Financial Services Organizations Can Help Ensure Success with SOA," White Paper, SunGard, [Online], [Retrieved January 15, 2012], http://www.sungard.com/ /media/financ ialsystems/whitepapers/soa_white $\% 20$ pa per_howfinancialservicesorganizationscan helpensuresuccesswithsoa.ashx.

Yin, R. K. (2009). Case Study Research Design and Methods. CA: Sage Publications. 